\title{
The pumpless extracorporeal lung membrane provides complete respiratory support during complex airway reconstructions without inducing cellular trauma or a coagulatory and inflammatory response
}

\author{
David Sanchez-Lorente, MD, ${ }^{\mathrm{a}}$ Manuela Iglesias, MD, ${ }^{\mathrm{b}}$ Alberto Rodríguez, MD, ${ }^{\mathrm{a}}$ \\ Philipp Jungebluth, $\mathrm{MD},{ }^{\mathrm{c}}$ and Paolo Macchiarini, $\mathrm{MD}, \mathrm{PhD}^{\mathrm{c}}$
}

\begin{abstract}
Objective: Our objective was to investigate the capacity of a pumpless extracorporeal lung membrane (iLA) (Novalung; Novalung GmbH, Hechingen, Germany) to provide adequate respiratory support and the impact on morbidity/mortality during complex airway reconstruction.
\end{abstract}

\begin{abstract}
Methods: Only patients unable to be ventilated via conventional intubation were eligible for the study. A larynx mask or orotracheal tubes were placed above the airway defect and the iLA was attached via femoral vessels (arteriovenous), providing extracorporeal gas exchange, apneic hyperoxygenation, and total tubeless airway reconstruction. Haptoglobulin, plasmin-antiplasmin complex, P-selectin activation, and interleukin 6 were measured before, during, and after iLA use and 72 hours postoperatively.

Results: Fifteen consecutive patients (age, $42 \pm 17$ years) underwent elective $(n=7)$ or emergency $(n=8)$ reconstruction of the airway owing to a variety of disorders or defects. The iLA was left in place for $185 \pm 61$ minutes, diverted $1.70 \pm 0.48 \mathrm{~L} / \mathrm{min}$ of the cardiac output, and provided an arteriovenous carbon dioxide removal and oxygen transfer of $173 \pm 94$ and $144 \pm 83 \mathrm{~mL} / \mathrm{min}$, respectively. The arterial oxygen tension/inspired oxygen fraction $(314 \pm 31 \mathrm{~mm} \mathrm{Hg})$, and arterial carbon dioxide tension $(40 \pm 6 \mathrm{~mm} \mathrm{Hg})$ remained stable throughout the entire operations. The following procedures were performed: redo slide tracheoplasties $(\mathrm{n}=3)$, redo tracheoesophageal fistula repair $(\mathrm{n}=1)$, sleeve lobectomies $(\mathrm{n}=2)$, main carina reconstructions $(\mathrm{n}=7)$, and anastomotic stenting and myocutaneous coverages $(\mathrm{n}=2)$. Three patients required prolonged ( $9 \pm 2$ days) postoperative iLA support. Two (13\%) patients died during the hospital stay. The use of iLA was associated with significant $(P<.05)$ but clinically nonrelevant and yet nonpathologic increases of haptoglobulin (hemolysis), plasmin-antiplasmin complex (coagulation activation), and P-selectin activation (platelet activation). Data normalized within 48 hours postoperatively.
\end{abstract}

Conclusions: Data suggest that iLA provides complete intraoperative respiratory support in patients who cannot receive conventional intubation/ventilation without relevant effects on cellular trauma, coagulatory response, and inflammatory response. (J Thorac Cardiovasc Surg 2012;144:425-30)

Surgical resection remains the only potential curative option for a variety of thoracic malignant diseases. Some advanced thoracic afflictions, particularly in cases of airway involvement, cannot be safely resected using conventional ventilation techniques. ${ }^{1,2}$ Cardiopulmonary bypass (CPB) has been described as an alternative to achieve reasonable oxygenation during surgical resection ${ }^{3,4}$; however, the use

\footnotetext{
From the Department of General Thoracic Surgery Hospital Clínic, ${ }^{a}$ Universitat de Barcelona, Spain; the Department of General Thoracic Surgery, ${ }^{b}$ Hospital Universitari Mutua de Terrassa, Spain; and the Advanced Center for Translational Regenerative Medicine (ACTREM), ${ }^{\mathrm{c}}$ Karolinska Institutet, Stockholm, Sweden.

Disclosures: Authors have nothing to disclose with regard to commercial support.

Received for publication Nov 29, 2011; revisions received March 9, 2012; accepted for publication April 3, 2012; available ahead of print May 11, 2012.

Address for reprints: Paolo Macchiarini, MD, PhD, Advanced Center for Translational Regenerative Medicine (ACTREM), European Airway Institute, Division of Ear, Nose and Throat (CLINTEC), Karolinska Institutet, Alfred Nobels Allé 8, Huddinge, SE-141 86 Stockholm (E-mail: paolo.macchiarini@ki.se). $0022-5223 / \$ 36.00$

Copyright (c) 2012 by The American Association for Thoracic Surgery doi:10.1016/j.jtcvs.2012.04.002
}

of CPB is associated with several disadvantages, such as increased bleeding, extensive blood administration, immunosuppressive effect, or tumor cell dissemination, all resulting in increased morbidity and mortality., 3,6

A relatively new extracorporeal pumpless lung membrane support, the interventional lung assist (iLA) (Novalung; Novalung GmbH, Hechingen, Germany), has been widely described and evaluated in experimental and clinical settings as a safe and efficient device, allowing near-total carbon dioxide removal and moderate oxygenation with minimal cellular trauma, coagulation alterations, and immunologic response. ${ }^{7-11}$

This study was conducted to evaluate the feasibility and efficacy of using the pumpless iLA intraoperatively in patients with thoracic diseases affecting the airways. These patients could not be managed with a standard intubation/ ventilation protocol to perform curative resection and reconstruction owing to clinical conditions or anatomic difficulties. 


\section{Abbreviations and Acronyms \\ ARDS = acute respiratory distress syndrome \\ $\mathrm{AVCO}_{2} \mathrm{R}=$ arteriovenous extracorporeal carbon dioxide removal \\ $\mathrm{CPB}=$ cardiopulmonary bypass \\ ECMO = extracorporeal membrane oxygenator \\ $\mathrm{FIO}_{2} \quad=$ fraction of inspiratory oxygen \\ iLA $=$ interventional lung assist \\ $\mathrm{PaCO}_{2}=$ arterial carbon dioxide tension \\ $\mathrm{PaO}_{2}=$ arterial oxygen tension}

\section{PATIENTS AND METHODS}

From January 1, 2005, to May 1, 2008, fifteen patients took part in our prospective study. All patients required elective or emergency complex airway reconstructions and could not be intubated in standard or selective procedures and thus related insufficient mechanical ventilation. Several parameters were evaluated to study the efficacy and feasibility of using the iLA. Adequacy of ventilation was controlled via blood gases, and mechanical and systemic effects of the extracorporeal device were measured by red blood cell damage, fibrinolysis, coagulation activation, and cytokine expression. The study was approved by the local institutional review board and the Ethics Committee of the University of Barcelona. All patients gave their written informed consent before surgery. In case of emergency, informed consent was obtained from patients' surrogates.

\section{Eligibility Criteria}

Patients who required elective or emergency complex airway reconstructions were eligible for the study if standard or selective intubation and mechanical ventilation were technically or functionally impossible: These include patients with ventilator-induced lung injury or acute respiratory distress syndrome (ARDS) and severe clinical and respiratory conditions precluding 1-lung ventilation. Also included were patients with extensive airway defects requiring prolonged complex airway manipulation and reconstruction time for whom standard intermittent ventilation alone was not believed to be effective. Other eligibility criteria were (1) hemodynamic stability (mean arterial blood pressure $>60 \mathrm{~mm} \mathrm{Hg}$ with minimal vasoactive medication), (2) absence of peripheral arterial occlusive diseases to avoid impaired limb perfusion after cannulation, (3) no signs of heparininduced thrombocytopenia, or (4) active septic shock. The criteria for an ARDS followed the guidelines of the American-European Consensus Conference for ARDS. ${ }^{1,2}$

\section{Anesthetic and Intraoperative Management}

All patients were assessed via a preoperative study protocol including the medical history with physical examination, chest radiograph, hemogram and biochemical profile, electrocardiogram, and, if necessary, additional examinations for cardiologic high-risk patients, such as computed tomographic chest and cervical scan. Pulmonary function tests were performed if possible.

All patients undergoing elective procedures received a thoracic epidural catheter directly before surgical intervention to provide postoperative analgesia except in the presence of contraindications or the patient's refusal. Anesthesia was induced using propofol (Diprivan; AstraZeneca UK Limited, London, United Kingdom), remifentanil (Remifentanil Kern Pharma EFG; Terrassa, Barcelona, Spain), and cisatracurium (Nimvex; GSK, Madrid, Spain), and ceftriaxone 2 g (Rocefalin; Roche Pharma, Madrid, Spain) was administrated as prophylactic antibiotic therapy. The airway management was performed using an orotracheal tube placed bronchoscopically above the airway defect $(\mathrm{n}=11)$ or larynx mask $(n=4)$, combined with simultaneous insertion of the iLA device. Before the operation was performed, all patients were preoxygenated with an inspired oxygen fraction $\left(\mathrm{FiO}_{2}\right)$ of 1.0. If possible, both lungs were ventilated to maximize denitrogenation of the functional residual capacity and achieve a maximal oxygen tension. Moreover, this provides an adequate oxygenation during the apneic time period by an increase of intrapulmonary oxygen storage in the residual functional capacity.

Then, the airways were opened and prepared for reconstruction while care was taken to preserve and protect adjacent tissues and organs. During the reconstruction of the airways, a nasogastric tube or pediatric catheter was inserted into the distal airway to provide apneic hyperoxygenation via an oxygen flow of 12 to $15 \mathrm{~L} / \mathrm{min}$. Pulse oximetry was continuously monitored and arterial blood gases were intermittently monitored to mantain peripheral oxygen arterial saturation and partial arterial carbon dioxide at levels greater than $90 \%$ and 35 to $45 \mathrm{~mm} \mathrm{Hg}$, respectively.

Several blood samples were taken during the operation to study different blood markers, control arterial blood gases, and hemogram, ionogram, and renal function. Moreover, we monitored hemodynamic parameters, vasoactive drug administration, fluids or blood products, urine output, arterial oxygen tension $\left(\mathrm{PaO}_{2}\right)$ and arterial carbon dioxide tension $\left(\mathrm{PaCO}_{2}\right)$, period of iLA support, and surgical time point. We evaluated the capacity of the iLA to provide additional oxygen by calculating the oxygen content of blood entering and exiting the device and multiplying the difference by the flow rate through the device, according to the following formula:

$$
\begin{aligned}
\text { Oxygen transfer }(\mathrm{mL} / \mathrm{min})= & ([\text { Oxygen content as volume percent }] \\
& \times 10 \mathrm{dL} / \mathrm{L}) \times \text { iLA flow rate }(\mathrm{L} / \mathrm{min})
\end{aligned}
$$

Arteriovenous extracorporeal carbon dioxide removal $\left(\mathrm{AVCO}_{2} \mathrm{R}\right)$ provided by the iLA was calculated as follows:

$$
\begin{aligned}
\mathrm{AVCO}_{2} \mathrm{R}(\mathrm{mL} / \mathrm{min})= & \text { Carbon dioxide content in the inflow arterial } \\
& \text { cannula }- \text { Carbon dioxide content in the venous } \\
& \text { outflow cannula }(\mathrm{mL} \text { carbon dioxide } / \\
& 100 \mathrm{~mL} \text { blood }) \times \text { Blood flow iLA }(\mathrm{L} / \mathrm{min}) \times 10 .
\end{aligned}
$$

When the surgical procedure was completed we initiated weaning and iLA device removal in the majority of the patients, except 3 who required prolonged iLA support during intensive care unit stay.

\section{Management of the iLA}

Before the operation, an ultrasound study of femoral vessels was made to measure internal diameter of common femoral arteries and rule out vascular disease and venous abnormalities. The femoral artery (13F or $15 \mathrm{~F})$ and vein $(15 \mathrm{~F}$ or $17 \mathrm{~F})$ were then cannulated in the operating room by specially designed cannulas. Cannula size was dependent on the internal artery diameter (at least $20 \%$ smaller than the internal artery diameter to avoid limb ischemia). A venous cannula (one size bigger than the arterial cannula) was placed in the contralateral leg.

The cannulas were inserted using the Seldinger technique to avoid vessel trauma but assure that the guide wire, dilator, and cannulas could move freely. Then, cannulas were flushed out with a bolus of $50 \mathrm{~mL}$ of heparinized saline solution (heparin doses $10-20 \mathrm{IU} / \mathrm{kg}$ body weight) and clamped proximal. The iLA device was entirely deaired and filled with normal saline solution $(250 \mathrm{~mL}$ ) before cannulation. Before unclamping, all connections were secured and unclamping was performed progressively for 1 to 2 minutes to minimize impact on hemodynamics. The oxygen line was connected to the inflow port of the iLA, and an initial flow rate (sweep gas) of $1 \mathrm{~L} / \mathrm{min}$ was set up to provide a pressure gradient at the membrane level to enhance gaseous diffusion. At the time of tracheal reconstruction, we performed an apneic hyperoxygenation with removal of carbon dioxide through the iLA system. During the surgical procedure the gas exchange (oxygen and carbon dioxide) was monitored via blood 
samples (arterial side) and amount of carbon dioxide removal regulated with the sweep gas flow (between 1 and $15 \mathrm{~L} / \mathrm{min}$ ). The blood flow through the iLA device was monitored by a flow meter (Novalung; Emtec) placed at the outflow venous line. Cardiac output and arterial pressure were measured and fluids or vasoactive drugs (dopamine, norepinephrine) administrated if necessary. The arterial pressure was maintained at $60 \mathrm{~mm} \mathrm{Hg}$ or higher with a blood flow rate through the iLA of around $1 \mathrm{~L} / \mathrm{min}$ or higher. To assure sufficient peripheral circulation, we placed a pulse oximeter on the leg with the arterial cannula.

Sedation was determined at the operation's end point and weaning was induced according to standard ventilation protocols. Simultaneously, sweep gas flow was reduced progressively under the control of arterial blood gases. The iLA was typically removed when the arterial oxygen saturation was greater than $95 \%$ at an $\mathrm{FIO}_{2}$ of 0.5 or more, and a physiologic carbon dioxide measurement and decannulation were performed according to transcutaneous technique principles.

\section{Analyses of Plasma Markers}

Laboratory values of blood samples were taken from all patients and analyzed at the following time points: (1) before iLA implantation (baseline), (2) 10 minutes after initiating extrapulmonary ventilation, (3) 2 hours after starting the procedure (intraoperative), (4) after iLA removal, and (5) 72 hours after surgery. The following plasma markers were monitored owing to their respective impact during the extracorporeal period: tissue/ cell damage via the plasma levels of lactate dehydrogenase (reference values, 100-190 IU/L) and haptoglobin (reference values, 0.3-2.0 g/L) as a sign for hemolytic anemia (especially intravascular hemolysis); the activation of the fibrinolytic system via the determination of the plasma plasmin-antiplasmin complex (reference values, 120-700 $\mu \mathrm{g} / \mathrm{L}$ ); the activation of platelets and thus coagulation via the plasma P-selectin (Human P-Selectine/CD62P DuoSet ELISA Development Kit; R\&D Systems, Minneapolis, Minn); and inflammatory response via the plasma interleukin 6 (ELISA Kit; R\&D Systems) (reference values, $0.428-12.95 \mathrm{pg} / \mathrm{mL}$ ).

\section{Statistical Analysis}

Data collected are presented as mean \pm standard deviation and were analyzed using SPSS Statistical Package for Windows (SPSS 12.0; SPSS, Inc, Chicago, Ill). Data recorded during the procedure were compared with baseline data (pre-iLA). Continuous data were assessed for normality and tested using the 2-tailed paired sample Student test. Mortality was defined as death within 30 days of surgery.

\section{RESULTS}

The study included 15 consecutive patients (Table 1) ( 9 male and 6 female) with a median age of $42 \pm 17$ years who underwent complex reconstruction of the airway using extracorporeal pumpless membrane support. Seven patients were managed under elective and 8 under emergency conditions. Three patients were already intubated at the time of surgery owing to respiratory disorders and 12 had elective procedures. Indications for surgery differed as follows: (1) unsuccessful supracarinal tracheal resection $(\mathrm{n}=4)$, (2) chronic tracheoesophageal benign fistula $(\mathrm{n}=1)$, (3) postcarinal pneumonectomy anastomotic dehiscence $(\mathrm{n}=2)$, (4) lung mass in a woman who was 6 months pregnant $(\mathrm{n}=1),(5)$ complete loss of tracheobronchial membranous wall $(n=4)$, and (6) main carinal perforation $(n=3)$. The mean ratio of partial pressure of arterial oxygen $\left(\mathrm{PaO}_{2}\right)$ to the fraction of inspired oxygen $\left(\mathrm{FiO}_{2}\right)\left(\mathrm{PaO}_{2} / \mathrm{FIO}_{2}\right.$
TABLE 1. Characteristics of the patients

\begin{tabular}{lc}
\hline \multicolumn{1}{c}{ Characteristics } & Numbers \\
\hline Gender (male vs female) & $9 / 6$ \\
Age (y) & $42 \pm 17$ \\
Type of surgery (elective/emergency) & $7 / 8$ \\
Indications & \\
Failed supracarinal tracheal resection & 4 \\
Chronic tracheoesophageal benign fistula & 1 \\
Postcarinal pneumonectomy anastomotic dehiscence & 2 \\
Lung mass in a woman who was 6 m pregnant & 1 \\
Complete loss of the tracheobronchial membraneous wall & 4 \\
Main carinal perforation & 3 \\
\hline
\end{tabular}

ratio) preoperatively was $196 \pm 71 \mathrm{~mm} \mathrm{Hg}$ and all except the 3 preoperatively intubated patients had an epidural catheter.

The iLA (Table 2) was inserted in the operating room into all patients before the operation according to the technique previously described without any complications. The device was left in place for $185 \pm 61$ minutes with a median flow of $1.70 \pm 0.48 \mathrm{~L} / \mathrm{min}$ and allowed an oxygen transfer and carbon dioxide removal $\left(\mathrm{AVCO}_{2} \mathrm{R}\right)$ of $144 \pm 83 \mathrm{~mL} /$ min and $173 \pm 94 \mathrm{~mL} / \mathrm{min}$, respectively; consequently, the respiratory settings of the mechanical ventilation during iLA support could be significantly decreased to nearly static ventilation (tidal volume, $2.4 \pm 0.5 \mathrm{~mL} / \mathrm{kg}$; respiratory rate, $6 \pm 1$ breaths/min; positive end-expiratory pressure, $12.3 \pm$ $2.6 \mathrm{~cm} \mathrm{H} \mathrm{H}_{2} \mathrm{O}$ ), maintaining a $\mathrm{PaO}_{2} / \mathrm{FIO}_{2}$ and $\mathrm{PaCO}_{2}$ of $314 \pm$ $31 \mathrm{~mm} \mathrm{Hg}$ and $40 \pm 6 \mathrm{~mm} \mathrm{Hg}$, respectively, avoiding an increase of respiratory damage owing to the mechanical ventilation, and allowing an extended apneic oxygenation period for the airway reconstruction when the mechanical ventilation was stopped. The mean time of apneic oxygenation needed mainly during airway reconstruction after dissection was $36 \pm 8$ minutes.

The operations performed in this series included the following: redo slide tracheoplasties $(\mathrm{n}=3)$, redo

TABLE 2. Respiratory variables during intraoperative extracorporeal lung membrane support

\begin{tabular}{lc}
\hline \multicolumn{1}{c}{ Variables } & iLA support \\
\hline Time $(\mathrm{min})$ & $185 \pm 61$ \\
Tidal volume (mL/kg) & $2.4 \pm 0.5$ \\
Respiratory frequency (beats/min) & $6 \pm 1$ \\
$\mathrm{FIO}_{2}$ & $0.5 \pm 0.2$ \\
$\mathrm{PaO}_{2} / \mathrm{FIO}_{2}(\mathrm{~mm} \mathrm{Hg})$ & $314 \pm 31$ \\
$\mathrm{PaCO}_{2}(\mathrm{~mm} \mathrm{Hg})$ & $40 \pm 6$ \\
$\mathrm{AVCO}_{2} \mathrm{R}(\mathrm{mL} / \mathrm{min})$ & $173 \pm 94$ \\
Oxygen transfer $(\mathrm{mL} / \mathrm{min})$ & $144 \pm 83$ \\
$\mathrm{PEEP}\left(\mathrm{cm} \mathrm{H} \mathrm{H}_{2} \mathrm{O}\right)$ & $12.3 \pm 2.6^{*}$ \\
\hline
\end{tabular}

$i \mathrm{iLA}$, Interventional lung assist; $\mathrm{Fio}_{2}$, fraction of inspiratory oxygen; $\mathrm{Pao}_{2}$, arterial oxygen tension; $\mathrm{PaCO}_{2}$, arterial carbon dioxide tension; $\mathrm{AVCO}_{2} \mathrm{R}$, arteriovenous carbon dioxide removal; PEEP, positive end-expired pressure. *During the period of no open airway (near static ventilation). There was no PEEP during open airway and apneic oxygenation. 


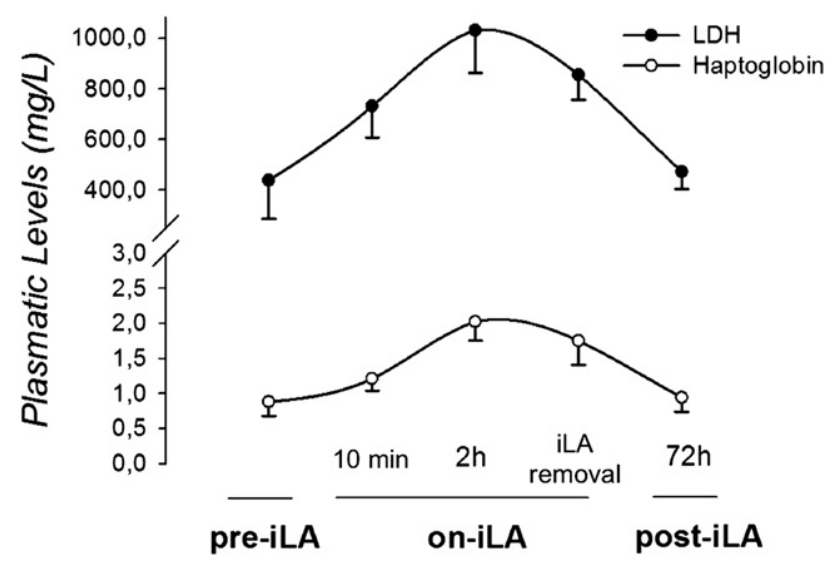

FIGURE 1. Dynamic of lactate dehydrogenase $(L D H)$ and haptoglobin level before interventional lung assist (iLA) placement (pre-iLA), during iLA function (on-iLA: 10 minutes and 2 hours after initiating iLA, and iLA removal), and after iLA removal (post-iLA).

tracheoesophageal fistula repair $(\mathrm{n}=1)$, sleeve lobectomies $(\mathrm{n}=2)$, main carina reconstructions $(\mathrm{n}=7)$, and anastomotic stenting and myocutaneous coverages $(n=2)$. The iLA was removed and decannulation performed according to transcutaneous technique principles during the first postoperative hour in all but 3 patients who had ventilator-induced lung injury/ARDS and therefore required prolonged iLA support ( $9 \pm 2$ days) during the intensive care unit stay. Two $(13 \%)$ patients died during the hospital stay, both of ARDS refractory to protective ventilation and iLA support. The mechanical ventilation duration was $5 \pm 4$ days, length of intensive care unit stay $8 \pm 3$ days, and total hospital stay $21 \pm 6$ days.

During the iLA period, several plasma markers increased significantly $(P<.05)$ : lactate dehydrogenase (Figure 1$)$, as a sign for hemolysis, as well as plasmin-antiplasmin

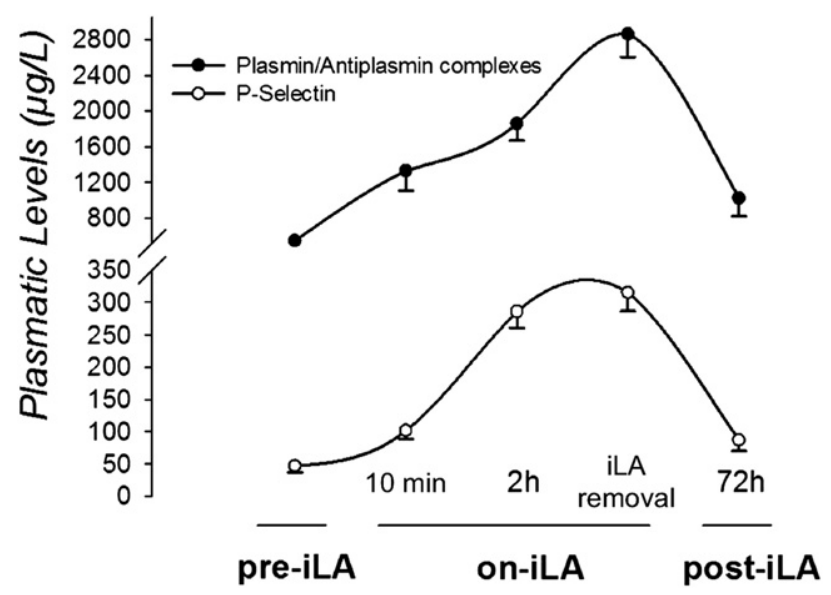

FIGURE 2. Dynamic of coagulation activation (plasmin-antiplasmin complex) and platelet activation (P-selectin) level before interventional lung assist (iLA) placement (pre-iLA), during iLA function (on-iLA: 10 minutes and 2 hours after initiating iLA, and iLA removal), and after iLA removal (post-iLA).

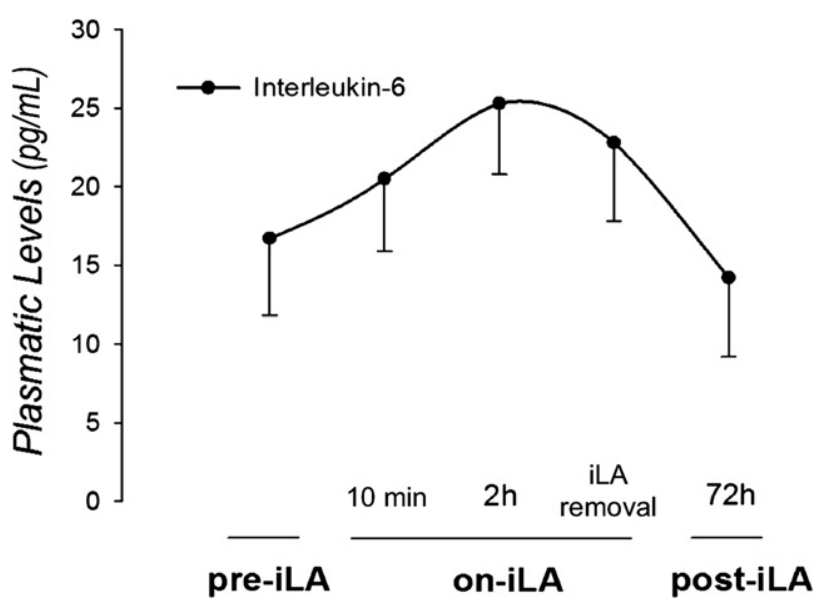

FIGURE 3. Dynamic inflammatory response (interleukin 6) level before interventional lung assist ( $i L A$ ) placement (pre-iLA), during iLA function (on-iLA: 10 minutes and 2 hours after initiating iLA, and iLA removal), and after iLA removal (post-iLA).

complex and P-selectin activation, as signs for coagulation and platelets activation, respectively (Figure 2). Moreover, inflammatory activation was detected via interleukin 6 upregulation (Figure 3). Even though these changes were statistically significant, none of the parameters reached pathologic levels within or after the iLA period. Besides, alterations showed no clinical relevance and normalized within 48 hours after iLA removal.

Morbidity related to the iLA included a femoral arterial thrombosis (1 patient) and dissection of the cannulated femoral arterial (1 patient) caused by the iLA catheter insertion. Both complications required surgical intervention after iLA removal.

\section{DISCUSSION}

The management of patients with extended airway defects/disorders continues to be clinically challenging. In cases that include extensive tracheal lesions for which conventional resection with end-to-end anastomosis cannot be considered, different techniques and solutions have been described. ${ }^{12-14}$ Yet, postoperative mortality and morbidity are rather common and related to intraoperative airway manipulation and the use of high-pressure ventilation. ${ }^{15}$ Increased ventilation settings result in epithelial irritation, inflammation, suture stress, and direct trauma of the newly reconstructed airway. Moreover, complex interventions on the airways are associated with difficulties to maintain adequate ventilation. The interruption of mechanical ventilation during the reconstruction, in particular, results in deficient oxygenation, insufficient ventilation (carbon dioxide removal), and the development of atelectasis. ${ }^{16,17}$ Therefore, an alternative to provide sufficient ventilation during complex airway operations is absolutely required. Different alternatives have been implemented. The oldest 
option in this context is the use of CPB. The use of CPB has been described in several articles with good surgical results. Unfortunately high complications rates (eg, coagulation and platelet alteration, immunosuppression, extensive bleeding, and dissemination of tumoral cells) lead to increased morbidity and mortality levels during and after the use of $\mathrm{CPB}^{3-5}$ During the past decades, the extracorporeal membrane oxygenator (ECMO) has been described as an alternative to $\mathrm{CPB}$ in resections of extensive malignant disease not affecting the heart, aortic arch, or pulmonary trunk. ECMO is a method that uses a venoarterial or a venovenous system and may become the standard device for extracorporeal lung assist therapy owing to the better results regarding mortality and morbidity compared with CPB. However, associated complications such as thromobocytopenia, thromoboembolic events, bleeding complications, and capillary leak syndromes remain prevalent, and lung injury and multiorganic failure are frequently encountered. ${ }^{18,19}$ Furthermore, the complex equipment requires a well-trained medical staff in dedicated units to guarantee adequate management of ECMO therapy.

Using the iLA in combination with an apneic ventilation protocol, our data provide evidence of optimal gas exchange during complex airway reconstruction (Table 2). Apneic hyperoxygenation (including preoxygenation and hyperventilation with 1.0 oxygen) allows for total apnea but adequate oxygenation for 10 to 20 minutes during airway operations. ${ }^{20,21}$ This apneic period is dramatically reduced in patients with pulmonary disease. Another crucial factor is the dramatic increase of $\mathrm{PaCO}_{2}$ during the apneic period owing to a lack of ventilation. Using the extracorporeal pumpless iLA could increase the apneic time (mean time in our study, $36 \pm 8$ minutes) to perform airway reconstruction for this distinct patient cohort without increasing the intraoperative risks, and this was recently confirmed by Wiebe and associates. ${ }^{22}$

ECMO-associated problems such as blood cell trauma and systemic inflammatory response are caused by the use of blood pumps and blood-material interactions. ${ }^{23,24}$ These complications can be minimized by using a pumpless device like the iLA. Besides, compared with ECMO, the small extracorporeal surface area of the iLA reduces the blood-material platelet activation significantly. The small surface avoids the need for high blood volumes or specific heater installations to stabilize blood temperature. In addition, less cell trauma and inflammatory response are detected. Experimental and clinical data support the concept that the iLA device causes minimal cell trauma and inflammatory response. ${ }^{7,8,18}$ Parameters associated with tissue/cell damage, activation of the fibrinolytic system, activation of the coagulation, and inflammatory response (Figures 1 to $3)$ showed a significant $(P>.05)$ increase in our study during the iLA period. The findings may promote the assumption that the use of the iLA may be contraindicated for this specific patient cohort. However, all described parameters remained within the physiologic range, did not reach pathologic levels, and revert rapidly after iLA removal back to baseline values. Notably, the total iLA period coincided with the span of surgery time. The described increase of parameters correlates directly with the complexity of the surgical procedure.

In conclusion, our results suggest that the pumpless extracorporeal lung membrane (iLA) is a very effective and promising strategy in patients who cannot be managed with a standard intubation and mechanical ventilation. The device provides a complete intraoperative respiratory support when combined with an apneic ventilation protocol, and this will prolong the period of surgical intervention of the airways. The avoidance of significant cellular trauma and coagulatory and inflammatory response represents the basic principle of the concept and allows for safe airway reconstruction without increased risks of perioperative and postoperative morbidity and mortality. More patients must be managed with this extracorporeal lung membrane device before definite predications for potential guidelines of using this method can be made.

\section{References}

1. Artigas A, Bernard GR, Carlet J, Dreyfuss D, Gattinoni L, Hudson L, et al. The American-European Consensus Conference on ARDS, part 2: ventilatory, pharmacologic, supportive therapy, study design strategies, and issues related to recovery and remodeling. Acute respiratory distress syndrome. Am J Respir Crit Care Med. 1998;157(4 Pt 1):1332-47.

2. Bernard GR, Artigas A, Brigham KL, Carlet J, Falke K, Hudson L, et al. The American-European Consensus Conference on ARDS. Definitions, mechanisms, relevant outcomes, and clinical trial coordination. Am J Respir Crit Care Med. 1994;149(3 Pt 1):818-24.

3. Wiebe K, Baraki H, Macchiarini P, Haverich A. Extended pulmonary resections of advanced thoracic malignancies with support of cardiopulmonary bypass. Eur J Cardiothorac Surg. 2006;29:571-7; discussion 577-8.

4. Ulicny KS Jr, Schmelzer V, Flege JB Jr, Todd JC, Mitts DL, Melvin DB, et al. Concomitant cardiac and pulmonary operation: the role of cardiopulmonary bypass. Ann Thorac Surg. 1992;54:289-95.

5. Hasegawa S, Otake Y, Bando T, Cho H, Inui K, Wada H. Pulmonary dissemination of tumor cells after extended resection of thyroid carcinoma with cardiopulmonary bypass. J Thorac Cardiovasc Surg. 2002;124:635-6.

6. McBride WT, Armstrong MA, Crockard AD, McMurray TJ, Rea JM. Cytokine balance and immunosuppressive changes at cardiac surgery: contrasting response between patients and isolated CPB circuits. Br J Anaesth. 1995;75:724-33.

7. Iglesias M, Jungebluth P, Petit C, Matute MP, Rovira I, Martinez E, et al. Extracorporeal lung membrane provides better lung protection than conventional treatment for severe postpneumonectomy noncardiogenic acute respiratory distress syndrome. J Thorac Cardiovasc Surg. 2008;135:1362-71.

8. Iglesias M, Martinez E, Badia JR, Macchiarini P. Extrapulmonary ventilation for unresponsive severe acute respiratory distress syndrome after pulmonary resection. Ann Thorac Surg. 2008;85:237-44; discussion 244.

9. Johnson P, Frohlich S, Westbrook A. Use of extracorporeal membrane lung assist device (Novalung) in H1N1 patients. J Card Surg. 2011;26:449-52.

10. Hommel M, Deja M, von Dossow V, Diemel K, Heidenhain C, Spies C, et al Bronchial fistulae in ARDS patients: management with an extracorporeal lung assist device. Eur Respir J. 2008;32:1652-5.

11. Walles T, Steger V, Wurst H, Schmidt KD, Friedel G. Pumpless extracorporeal gas exchange aiding central airway surgery. J Thorac Cardiovasc Surg. 2008;136:1372-4.

12. Grillo HC, ed. Surgery of trachea and bronchi. Hamilton: BC Decker; 2004. p. 518-47.

13. Garabedian EN, Le Bret E, Corre A, Roger G, Pineau E, Bourel P, et al. Tracheal resection associated with slide tracheoplasty for long-segment congenital 
tracheal stenosis involving the carina. J Thorac Cardiovasc Surg. 2001;121: 393-5.

14. Macchiarini P, Altmayer M, Go T, Walles T, Schulze K, Wildfang I, et al. Interdisciplinary Intrathoracic Tumor Task Force. Technical innovations of carinal resection for nonsmall-cell lung cancer. Ann Thorac Surg. 2006;82:1989-97; discussion 1997.

15. Idriss FS, DeLeon SY, Ilbawi MN, Gerson CR, Tucker GF, Holinger L. Tracheoplasty with pericardial patch for extensive tracheal stenosis in infants and children. J Thorac Cardiovasc Surg. 1984;88:527-36.

16. Karzai W, Schwarzkopf K. Hypoxemia during one-lung ventilation: prediction, prevention, and treatment. Anesthesiology. 2009;110:1402-11.

17. Ishikawa S, Lohser J. One-lung ventilation and arterial oxygenation. Curr Opin Anaesthesiol. 2011;24:24-31.

18. Chalwin RP, Moran JL, Graham PL. The role of extracorporeal membrane oxygenation for treatment of the adult respiratory distress syndrome: review and quantitative analysis. Anaesth Intensive Care. 2008;36:152-61.
19. Morris AH, Wallace CJ, Menlove RL, Clemmer TP, Orme JF Jr, Weaver LK, et al. Randomized clinical trial of pressure-controlled inverse ratio ventilation and extracorporeal $\mathrm{CO} 2$ removal for adult respiratory distress syndrome. Am J Respir Crit Care Med. 1994;149(2 Pt 1):295-305.

20. Frumin MJ, Epstein RM, Cohen G. Apneic oxygenation in man. Anesthesiology. 1959;20:789-98.

21. Fraioli RL, Sheffer LA, Steffenson JL. Pulmonary and cardiovascular effects of apneic oxygenation in man. Anesthesiology. 1973;39:588-96.

22. Wiebe K, Poeling J, Arlt M, Philipp A, Camboni D, Hofmann S, et al. Thoracic surgical procedures supported by a pumpless interventional lung assist. Ann Thorac Surg. 2010;89:1782-7.

23. Upp JR Jr, Bush PE, Zwischenberger JB. Complications of neonatal extracorporeal membrane oxygenation. Perfusion. 1994;9:241-56.

24. Asimakopoulos G, Smith PL, Ratnatunga CP, Taylor KM. Lung injury and acute respiratory distress syndrome after cardiopulmonary bypass. Ann Thorac Surg. 1999;68:1107-15. 